\title{
Long Non-Coding RNA LUCAT1 Promotes Proliferation and Invasion in Clear Cell Renal Cell Carcinoma Through AKT/GSK-3 $\beta$ Signaling Pathway
}

\author{
Zaosong Zhenga,b Fengjin Zhao ${ }^{\mathrm{a}}$ Dingjun Zhu ${ }^{\mathrm{a}}$ Jinli Han ${ }^{\mathrm{a}}$ Haicheng Chen \\ Yuhong Cai ${ }^{a}$ Zhiliang Chen ${ }^{a}$ Wenlian Xie ${ }^{a}$ \\ aDepartment of Urology, Sun Yat-sen Memorial Hospital, Sun Yat-sen University, Guangzhou, \\ ${ }^{b}$ Guangdong Provincial Key Laboratory of Malignant Tumor Epigenetics and Gene Regulation, Sun Yat- \\ sen Memorial Hospital, Sun Yat-sen University, Guangzhou, China
}

\section{Key Words}

Lncrna $\bullet$ LUCAT1 $\bullet$ CXCL2 $・$ Clear cell renal cell carcinoma $\bullet$ Proliferation $\bullet$ Invasion

\begin{abstract}
Background/Aims: Long non-coding RNAs (IncRNAs) have emerged as new regulators and biomarkers in several cancers. However, few IncRNAs have been well characterized in clear cell renal cell carcinoma (ccRCC). Methods: We investigated the IncRNA expression profile by microarray analysis in 5 corresponding $\mathrm{CCRCC}$ tissues and adjacent normal tissues. Lung cancer-associated transcript 1 (LUCAT1) expression was examined in 90 paired ccRCC tissues by real-time PCR and validated by The Cancer Genome Atlas (TCGA) database. Kaplan-Meier analysis was used to examine the prognostic value of LUCAT1 and CXCL2 in CCRCC patients. Loss and gain of function were performed to explore the effect of LUCAT1 on proliferation and invasion in CCRCC cells. Western blotting was performed to evaluate the underlying mechanisms of LUCAT1 in cCRCC progression. Chemokine stimulation assay was performed to investigate possible mechanisms controlling LUCAT1 expression in cCRCC cells. Enzyme-linked immunosorbent assays were performed to determine serum CXCL2 in cCRCC patients and healthy volunteers. Receiver operating characteristic curve analysis was performed to examine the clinical diagnostic value of serum CXCL2 in CCRCC. Results: We found that LUCAT1 was significantly upregulated in both clinical ccRCC tissues $(n=90)$ and TCGA ccRCC tissues $(n=$ 448) compared with normal tissues. Statistical analysis revealed that the LUCAT1 expression level positively correlated with tumor T stage $(P<0.01)$, M stage $(P<0.01)$, and TNM stage $(P<0.01)$. Overall survival and disease-free survival time were significantly shorter in the high-LUCAT1-expression group than in the low-LUCAT1-expression group (log-rank $P<0.01$ ). LUCAT1 knockdown inhibited ccRCC cell proliferation and colony formation, induced cell cycle arrest at G1 phase, and inhibited cell migration and invasion. Overexpression of LUCAT1 Z. Zheng and F. Zhao contributed equally to this work.


promoted proliferation, migration, and invasion of ccRCC cells. Mechanistic investigations showed that LUCAT1 induced cell cycle G1 arrest by regulating the expression of cyclin D1, cyclin-dependent kinase 4, and phosphorylated retinoblastoma transcriptional corepressor 1. Moreover, LUCAT1 promoted proliferation and invasion in CCRCC cells partly through inducing the phosphorylation of AKT and suppressing the phosphorylation of GSK-3 $\beta$. We also revealed that chemokine CXCL2, upregulated in CCRCC, induced LUCAT1 expression and might be a diagnostic and prognostic biomarker in CCRCC. Conclusions: LUCAT1 was upregulated in ccRCC tissues and renal cancer cell lines, and significantly correlated with malignant stage and poor prognosis in cCRCC. LUCAT1 promoted proliferation and invasion in cCRCC cells through the AKT/GSK-3 $\beta$ signaling pathway. We also revealed that LUCAT1 overexpression was induced by chemokine CXCL2. These findings indicate that the CXCL2/LUCAT1/AKT/GSK$3 \beta$ axis is a potential therapeutic target and molecular biomarker for ccRCC.

\section{Introduction}

C 2018 The Author(s)

Published by S. Karger AG, Basel

Renal cell carcinoma (RCC) is one of the ten most common cancers, and its incidence and mortality have increased for over two decades [1]. Among the different histological types, clear cell RCC (ccRCC) is the most common subtype and accounts for approximately $70-75 \%$ of RCC [2]. Despite the widespread use of imaging in examinations, about $17 \%$ of all RCCs have distant metastases at the time of diagnosis and about $30 \%$ of patients treated for localized RCC will relapse [3,4]. Median survival for RCC patients with metastatic disease is about 13 months [5]. However, the molecular mechanisms of RCC proliferation and invasion are still unclear. Thus, exploring possible molecular mechanisms holds great promise in management of RCC.

The discovery of non-coding RNAs (ncRNAs) in the human genome was a remarkable breakthrough in the post-genome era. Long ncRNAs (lncRNAs), one important subtype of ncRNAs, are more than 200 nucleotides long but lack protein-coding capacity [6]. Extensive research indicates that lncRNAs are aberrantly expressed in many human cancers and have comprehensive function in cancer proliferation and metastasis [7]. For example, the lncRNA metastasis-associated lung adenocarcinoma transcript 1 has been shown to be involved in proliferation and metastasis of several cancer subtypes, such as lung cancer, renal cancer, and pancreatic cancer [8-10]. The IncRNA HOX transcript antisense RNA has been shown to promote metastasis by interacting with polycomb repressive complex 2 and predict poor prognosis in breast cancer [11]. Studies have revealed that some aberrantly expressed lncRNAs are significantly associated with the prognosis of cancers, such as the lncRNAs HOXA transcript at the distal tip in hepatocellular carcinoma [12], plasmacytoma variant translocation 1 in gastric cancer [13], and urothelial carcinoma associated 1 in bladder cancer [14]. However, there has been relatively little research on the role of lncRNAs in ccRCC, and the overall contribution of IncRNAs to ccRCC remains largely unknown.

In this study, we performed microarray analysis to investigate the lncRNAs and proteincoding gene expression profile of ccRCC using 5 corresponding ccRCC tissues and adjacent normal tissues. The IncRNA lung cancer-associated transcript 1 (LUCAT1) [15], one of the most upregulated lncRNAs, was overexpressed in ccRCC tissues and renal cancer cell lines and significantly correlated with malignant stage and poor prognosis in ccRCC. Our findings indicated that LUCAT1 promoted proliferation, migration, and invasion of ccRCC cells. LUCAT1 induced cell cycle G1 arrest by regulating the expression of cyclin D1, cyclin-dependent kinase 4 (CDK4), and phosphorylated retinoblastoma transcriptional corepressor $1(\mathrm{p}-\mathrm{Rb})$. Moreover, LUCAT1 promoted proliferation and invasion in ccRCC cells partly through the AKT/GSK-3 $\beta$ signaling pathway. We also revealed that LUCAT1 overexpression was induced by the chemokine CXCL2 in ccRCC. These results suggested that the CXCL2/LUCAT1/AKT/ GSK- $3 \beta$ axis promoted the progression of ccRCC, and might be a useful diagnostic biomarker and therapeutic target in ccRCC. 


\section{Cellular Physiology Cell Physiol Biochem 2018;48:891-904 and Biochemistry Published online: July 20, $2018 \quad$\begin{tabular}{l|l} 
DOI: 10.1159/000491957 2018 The Author(s). Published by S. Karger AG, Basel \\
www.karger.com/cpb
\end{tabular} Zheng et al.: LUCAT1 Promoted Proliferation and Invasion in Ccrcc}

\section{Materials and Methods}

\section{Microarray and computational analysis}

Total RNA from 5 pairs of ccRCC and matched adjacent normal tissues was extracted and used to synthesize double-stranded complementary DNA (cDNA). The cDNA was labeled and hybridized to the Human LncRNA Array V4.0 (CapitalBio Corp, Beijing, China) according to the manufacturer's instructions. The microarray data were analyzed for data summarization, normalization, and quality control using GeneSpring software v12 (Agilent, Santa Clara, CA). The data were log2-transformed and median-centered by gene using the Adjust Data function of Cluster 3.0 software. To select the differentially expressed genes, we used threshold values of $>2$ and $<-2$ fold change and a $P$ value $<0.05$. Hierarchical clustering was carried out on aberrantly expressed IncRNAs and mRNAs with Cluster 3.0 and Treeview software (Stanford University, Stanford, CA). All primary microarray data have been uploaded to the Gene Expression Omnibus (GEO): GSE96574.

The Cancer Genome Atlas ccRCC RNA-Seq and clinical data

The Cancer Genome Atlas (TCGA) database contains ccRCC RNA-Seq data and clinical data from 448 patients. We downloaded the RNA-Seq expression values and clinical information, including sex, age, tumor size, AJCC tumor pathologic pT, AJCC nodes pathologic pN, AJCC metastasis pathologic pM, AJCC pathologic tumor stage, overall survival status time, and disease-free time from TCGA data portal (https://tcga-data. nci.nih.gov/tcga/). We also downloaded RNA-Seq expression values for the selected lncRNA (LUCAT1) of 448 TCGA ccRCC patients and 67 matched normal samples from The Atlas of ncRNA in Cancer (TANRIC) database [16] (http://ibl.mdanderson.org/tanric/_design/basic/index.html).

\section{Patients and tissue samples}

A total of 90 pairs of ccRCC tissues and corresponding adjacent normal tissues were collected from patients who underwent radical or partial nephrectomy at Sun Yat-sen Memorial Hospital of Sun Yat-sen University. All tissue samples were stored in RNAlater at $-80^{\circ} \mathrm{C}$ until RNA extraction. All tissue samples were collected with written consent from patients and approved by the Hospital Ethics Review Committees. All samples were confirmed pathologically based on the eighth edition of the Tumor-Node-Metastasis Staging Classification for Urologic Cancers [17].

\section{Cell culture and RNA transfection}

The human RCC cell lines (786-0, Caki-1, Caki-2, ACHN, 769-P, and A-498) and one normal kidney cell line (HK-2) used in this study were obtained from American Type Culture Collection (ATCC, Manassas, VA). Caki-1 and Caki-2 cell lines were cultured in McCoy's 5a Modified Medium. 786-0 and 769-P were cultured in RPMI 1640 medium (Gibco BRL, Gaithersburg, MD). ACHN and A-498 cell lines were cultured in Dulbecco's modified Eagle's medium (Gibco). All media were supplemented with $10 \%$ fetal bovine serum (FBS; Hyclone Technologies, Logan, UT). All cell lines were maintained in a humidified incubator with 5\% $\mathrm{CO}_{2}$ at $37^{\circ} \mathrm{C}$. Small interfering RNAs (siRNAs) and lentivirus were obtained from Shanghai GenePharma (Shanghai, China) and siRNA transfection was conducted using Lipofectamine RNAiMAX Reagent (Life Technologies, Carlsbad, CA) according to the manufacturer's instructions.

\section{RNA extraction and quantitative real-time PCR}

Total RNA was extracted using TRIzol reagent (Life Technologies) and reversed transcribed into cDNA using PrimerScript RT Master (Takara Biotechnology (Dalian) Co., Ltd., Dalian, China). After reverse transcription of the total RNA, quantitative real-time PCR (RT-PCR) was conducted to examine the gene expression using SYBR-Green PCR Master mix (Roche, Mannheim, Germany) on a LightCycler 96 Real-Time PCR instrument (Roche). Relative gene expression level was determined using $\beta$-actin as a normalizer. Relative expression was calculated using the $2^{-\Delta \mathrm{CT}}$ method $(\Delta \mathrm{CT}=\mathrm{CT}$ value of $\beta$-actin $-\mathrm{CT}$ value of LUCAT1) and paired $t$ tests were used to compare gene expression levels. The primer sequences used in this study are listed in Table 1.

\section{Cell proliferation and colony formation assays}

Cell proliferation was measured using a CellTiter 96 AQueous One Solution Cell Proliferation Assay kit (Promega, Madison, WI). Cells $\left(10^{3}\right)$ were plated in each well of a 96-well plate and at least 5 replicate wells were used for each assay condition. Absorbance was measured with the multifunctional microplate 


\section{Cellular Physiology Cell Physiol Biochem 2018;48:891-904 \begin{tabular}{l|l} 
and Biochemistry Published online: July 20, 2018 & $\begin{array}{l}\text { (c) } 2018 \text { The Author(s). Published by S. Karger AG, Basel } \\
\text { www.karger.com/cpb }\end{array}$ \\
\hline
\end{tabular} Zheng et al.: LUCAT1 Promoted Proliferation and Invasion in Ccrcc}

reader SpectraMax M5 (Molecular Devices, San Jose, CA) at $490 \mathrm{~nm}$. The cell proliferation assay was conducted every $24 \mathrm{~h}$ and lasted 5 days. For the colony formation assay, $10^{3}$ cells were plated in each well of a 6 -well plate at 48 $\mathrm{h}$ after RNA transfection and incubated in the corresponding medium. After one week, cells were washed twice with phosphate-buffered saline (PBS), fixed with 4\% paraformaldehyde for $30 \mathrm{~min}$, and then stained with crystal violet for $30 \mathrm{~min}$ for visualization and counting. All assays were performed independently 3 times using triplicate wells.
Table 1. The sequences of primers and siRNAs

\begin{tabular}{lcc}
\hline & Forward (Sense) & Reverse (Antisense) \\
\hline$\beta$-actin & ACTGGAACGGTGAAGGTGAC & AGAGAAGTGGGGTGGCTTTT \\
TCONS_00010984 & AGATGGATAAACAGAGGCAACC & GCTCTTTATTTGTGAGGGGATG \\
TCONS_00010401 & CCACTCAGACAATGCCCAGAC & CAAGGTCCCATAAGAGTTCCAG \\
TCONS_00006755 & GCCAGTGTTTCACCTGTTTATC & CACTTACTGATTTCTCCAACG \\
ENST00000419921 & ACCGCACCCAGATACTTTTC & ACGCCTATAACCCAGCACAT \\
ENST00000502291 & TTTCAATGAGAAGCACAGGACC & GCAAGTAGACATGCCAGGAGA \\
TCONS_00018357 & AAGTTAAGGTCGCCGAAGTAG & GGAGATGATTTTGGGAGGG \\
TCONS_00011082 & GAATGAGGACAGAAAAGCACC & CCGATCAATGGCAAATACGA \\
ENST0000051225 & GAAGCCAGATGACTCCACAGT & AGGGGTAAGGGGAAAATAGG \\
LUCAT1 siRNA\#1 & CUCAGUGUCACACAUUUCATT & UGAAAUGUGUGACACUGAGTT \\
LUCAT1 siRNA\#2 & GAUUAAGUCACCUACUCAUTT & AUGAGUAGGUGACUUAAUCTT \\
\hline
\end{tabular}

Cell wound-healing, migration, and invasion assays

To evaluate the cell migration capacity, cell wound-healing and transwell migration assays were performed. For the cell wound-healing assay, cells were seeded into 12 -well plates at $48 \mathrm{~h}$ after RNA transfection. When cells reached $95-100 \%$ confluence, a wound was created by manually scraping the cell monolayer with a p200 pipet tip. The cells were then washed with PBS to remove cell debris and the medium replaced with serum-free medium. Images were captured by microscopy at 0 and $24 \mathrm{~h}$ after wounding. Transwell migration assay was performed by using transwell chamber inserts ( $8 \mu \mathrm{m}$ pore size; Corning Inc., Corning, NY). Approximately 104 cells were seeded into the upper chambers in serum-free medium. For transwell invasion assay, Matrigel Invasion Chambers $(8 \mu \mathrm{m})$ in 24-well plates (BD Bioscience, Franklin Lakes, NJ) were used and $2 \times 10^{5}$ cells were seeded into the upper chamber with serum-free medium. The lower chamber was filled with $600 \mu \mathrm{l}$ medium with 10\% FBS. After $24 \mathrm{~h}$, cells in upper chambers were removed, and cells migrating to or invading the lower surface of the membrane were fixed with $4 \%$ paraformaldehyde and stained with $0.1 \%$ crystal violet. The number of migrated or invaded cells was counted in 5 randomly selected fields under a microscope. All assays were performed independently 3 times.

\section{Flow cytometry assay}

Flow cytometry assay was performed to analyze the cell cycle and apoptosis of RCC cells. For cell cycle analysis, $2 \times 10^{5}$ cells were seeded into 6-well plates. At $48 \mathrm{~h}$ after siRNA transfection, cells were collected, washed with PBS, fixed with 70\% ice-cold ethanol overnight, and stained with propidium iodide (PI). The cell cycle was analyzed using a FACSVerse flow cytometer (Becton Dickinson, San Jose, CA). For the apoptosis assay, cells were harvested after 48-h transfection with siRNAs. The cells were stained with annexin V-FITC and PI, according to the manufacturer's instructions. Cell apoptotic rate was determined using the FACSVerse flow cytometer. All experiments were performed independently 3 times.

\section{Protein extraction and Western blotting}

Cells were washed 3 times with ice-cold PBS and lysed in RIPA buffer (Thermo Fisher Scientific, Waltham, MA) containing protease and phosphatase inhibitor cocktail (Roche). Total protein was extracted according to the manufacturer's instructions. The protein concentration was determined using the Bio-Rad assay system (Bio-Rad Laboratories, Hercules, CA). For Western blotting analysis, equal amounts of protein extract $(40 \mu \mathrm{g})$ were separated by electrophoresis in 10\% sodium dodecyl sulfate-polyacrylamide gels and the proteins were then transferred to polyvinylidene fluoride membranes (Merck Millipore, Burlington, MA). Blotted membranes were blocked in 5\% skim milk in TBST, followed by incubation with primary antibodies overnight at $4^{\circ} \mathrm{C}$. The membranes were then washed 3 times for $10 \mathrm{~min}$ each with TBST, and subsequently incubated $1 \mathrm{~h}$ with horseradish peroxidase-linked secondary antibody at room temperature. GAPDH was used as the internal control. The blots were detected using an enhanced chemiluminescence kit (Merck Millipore) with a Molecular Imager system (Bio-Rad Laboratories). All experiments were performed independently 3 times.

Enzyme-linked immunosorbent assay

The level of serum CXCL2 was detected using a human CXCL2 enzyme-linked immunosorbent assay (ELISA) kit (Neobioscience Technology, China) according to the manufacturer's instructions. The serum 


\section{Cellular Physiology Cell Physiol Biochem 2018;48:891-904

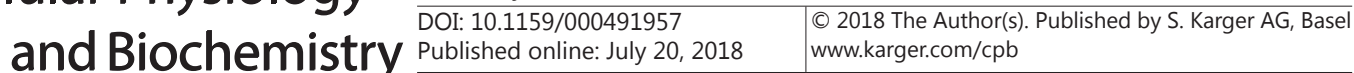 \\ Zheng et al.: LUCAT1 Promoted Proliferation and Invasion in Ccrcc}

samples of RCC patients and healthy volunteers were added into a 96-well plate, which was coated with anti-CXCL2 antibody, and incubated for $90 \mathrm{~min}$ at $37^{\circ} \mathrm{C}$. After washing 5 times with washing buffer, the secondary antibody was added into the plate and incubated for $60 \mathrm{~min}$ at $37^{\circ} \mathrm{C}$. The reactions were stopped and the results were analyzed with an ELISA reader (Multiskan Mk3, Thermo Fisher Scientific) at $450 \mathrm{~nm}$. Assays were performed independently 3 times using triplicate wells.

\section{Statistical analysis}

Statistical analyses were performed using GraphPad Prism (version 6.0; GraphPad Software, Inc., La Jolla, CA) and SPSS (version 10.0; SPSS Inc., Chicago, IL) with $P<0.05$ considered statistically significant. The significance of the differences between groups was analyzed using the Student's $t$ test or one-way analysis of variance (ANOVA). Overall survival and disease-free survival rates were calculated using the Kaplan-Meier method with the log-rank test for comparisons. The clinical diagnostic value of serum CXCL2 was verified by receiver operating characteristic (ROC) curve analysis.

\section{Results}

Microarray expression profiling identified differentially expressed lncRNAs and mRNAs in cCRCC tissues compared with adjacent normal tissues

To investigate the potential biological functions of transcriptional changes in ccRCC, we performed microarray analysis utilizing the Human LncRNA Array v4.0 targeting 40, 916 IncRNAs and 34, 235 protein-coding genes in 5 corresponding ccRCC tissues and adjacent normal tissues (GSE96574). Unsupervised hierarchical clustering was performed to determine the IncRNA and mRNA expression profiles in ccRCC and adjacent normal tissues (Fig. 1A and B). We then performed volcano-plot filtering: the red plots show the upregulated lncRNAs and the green plots show the downregulated ones (Fig. 1C). In total, we identified 1554 lncRNAs (943 upregulated and 611 downregulated) and 2504 mRNAs (1435 upregulated and 1069 downregulated) that were differentially expressed in ccRCC tissues with a fold change $>2$ and $P<0.01$, and the 10 most differentially expressed lncRNAs and mRNAs are listed in Table 2.

Fig. 1. Differential expression patterns of lncRNAs and mRNAs between ccRCC tissues and adjacent normal tissues (ANTs). (A) Heat map representing unsupervised hierarchical clustering of IncRNA expression levels in ccRCC tissues compared with ANTs. Each column represents the indicated tissue sample, and each row indicates one IncRNA. Red represents a higher expression level and green represents a lower expression level. (B) Heat map representing unsupervised hierarchical clustering of mRNA expression levels in ccRCC tissues compared with ANTs. (C) Volcano plots representing the variations of IncRNAs between ccRCC tissues and ANTs. (D and E) Validation of microarray analysis data in ccRCC tissues using 4 upregulated IncRNAs

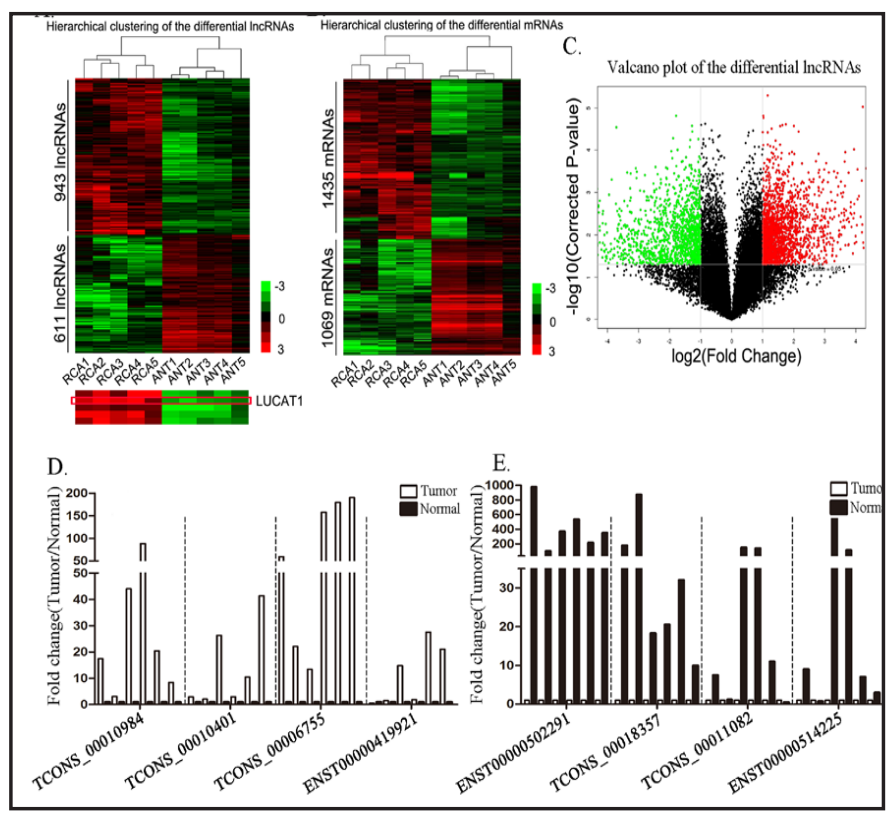
and 4 downregulated lncRNAs. ${ }^{*} \mathrm{P}<0.05$ by Student's t test.

\section{KARGER}


Validation of microarray data

In order to validate the microarray data and choose reliable lncRNAs for further analysis, 4 upregulated lncRNAs and 4 downregulated IncRNAs in the microarray analysis were selected and validated by RT-PCR. Consistent with the microarray data, RTPCR results showed that lncRNAs TCONS_00010984 and TCONS_00006755 were significantly upregulated, and lncRNAs ENST00000502291 and ENST00000301683 were significantly downregulated in ccRCC tissues compared with normal tissues (Fig. 1D and $\mathrm{E}$ ).

The IncRNA LUCAT1 is overexpressed in ccRCC

Next, we focused on lncRNA TCONS_00010984, officially named LUCAT1, for further study. We chose LUCAT1 for several reasons. Firstly, LUCAT1 was one of the most upregulated lncRNAs in microarray analysis and validation. Secondly, LUCAT1 expression positively correlated with aggressive stage in both the Sun Yat-sen cohort and TCGA cohort. Lastly, functional pre-experiments indicated that LUCAT1 acts as an oncogene in ccRCC.

To explore the role of LUCAT1 in ccRCC progression, we first examined LUCAT1 expression in 90 paired ccRCC tissues and adjacent normal tissues by RT-PCR. We found that LUCAT1 was upregulated in ccRCC tissues compared with adjacent normal tissues $(P<0.01)$ (Fig. 2A). Consistent with this result, LUCAT1 was found to be overexpressed in ccRCC tissues compared with normal tissues in
Table 2. Ten most upregulated and downregulated IncRNAs and mRNAs in ccRCC tissues compared with adjacent normal tissues

\begin{tabular}{lccccccc}
\hline IncRNA ID & IncRNAs & & & & mRNAs & \\
\hline ENST00000419921 & Fold change & P value & Regulation & Gene symbol & Fold change & P value & Regulation \\
TCONS_00010984 & 6.01 & 0.0069 & Up & CA9 & 7.50 & 0.0005 & Up \\
LIT3501 & 5.93 & 0.0002 & Up & CP & 6.64 & 0.0008 & Up \\
TCONS_00006755 & 5.90 & 0.0175 & Up & NPTX2 & 5.95 & 0.0073 & Up \\
TCONS_00010401 & 5.83 & 0.0027 & Up & PTHLH & 5.83 & 0.0044 & Up \\
HIT000332651 & 5.79 & 0.0003 & Up & NDUFA4L2 & 5.75 & 0.0001 & Up \\
NR_103549 & 5.70 & 0.0144 & Up & BHLHE41 & 5.47 & 0.0001 & Up \\
ENST00000456816 & 5.59 & 0.0009 & Up & FGG & 5.30 & 0.0231 & Up \\
ENST00000414120 & 5.47 & 0.0011 & Up & SAA1 & 5.18 & 0.0012 & Up \\
TCONS_00010991 & 5.46 & 0.0031 & Up & PRIMA1 & 5.18 & 0.0002 & Up \\
ENST00000439524 & 5.28 & 0.0023 & Up & INHBB & 5.15 & 0.0044 & Up \\
ENST00000514225 & 7.19 & 0.0024 & Down & ALDOB & 9.42 & 0.0005 & Down \\
ENST00000301683 & 7.10 & 0.0019 & Down & SLC22A8 & 9.08 & 0.0013 & Down \\
TCONS_00010550 & 6.82 & 0.0002 & Down & PAH & 8.72 & 0.0005 & Down \\
TCONS_00011082 & 6.79 & 0.0006 & Down & SLC12A1 & 8.68 & 0.0031 & Down \\
TCONS_00018357 & 6.61 & 0.0033 & Down & CRYAA & 8.67 & 0.0009 & Down \\
ENST00000502291 & 6.32 & 0.0030 & Down & SLC22A6 & 8.58 & 0.0010 & Down \\
ENST00000451776 & 6.26 & 0.0096 & Down & XPNPEP2 & 8.35 & 0.0011 & Down \\
ENST00000586145 & 6.10 & 0.0025 & Down & MT1G & 8.30 & 0.0004 & Down \\
ENST00000417193 & 6.10 & 0.0132 & Down & SLC36A2 & 7.80 & 0.0025 & Down \\
\hline & 5.97 & 0.0001 & Down & KNG1 & 7.63 & 0.0063 & Down \\
\hline
\end{tabular}

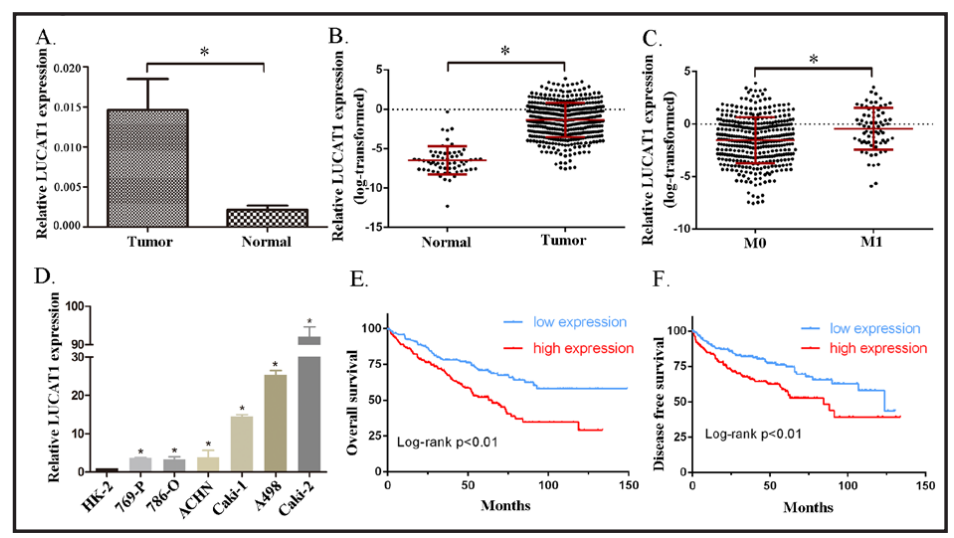

Fig. 2. LUCAT1 is frequently upregulated in ccRCC tissues and cell lines, acting as a negative prognostic factor. (A) Relative LUCAT1 expression in 90 pairs of ccRCC tissues and normal tissues (Student's $t$ test, ${ }^{*} \mathrm{P}<0.05$ ). (B) Validation of LUCAT1 expression in ccRCC tissues (n $=448)$ and normal tissues $(n=67)$ in TCGA database (Student's t test, $* \mathrm{P}<0.05)$. (C) Relative LUCAT1 expression in metastatic ccRCC tissues $(n=72)$ and non-metastatic ccRCC tissues $(n=376)$ in TCGA database (Student's t test, ${ }^{*} \mathrm{P}<0.05$ ). (D) Relative LUCAT1 expression in renal carcinoma cell lines (786-0, Caki-1, Caki-2, ACHN, 769-P, and A498) and a normal kidney cell line (HK-2) (ANOVA, $\left.{ }^{*} \mathrm{P}<0.05\right)$. ( $\mathrm{E}$ and $\mathrm{F}$ ) Kaplan-Meier overall survival curve and disease-free survival curve of patients with ccRCC in TCGA database according to LUCAT1 expression ( $\mathrm{n}=448$, log-rank $\mathrm{P}<0.01$ ). 
another independent cohort of 448 ccRCC samples and 67 normal samples available at TCGA database ( $\mathrm{P}<0.01$ ) (Fig. 2B and $\mathrm{C}$ ).

We further examined the expression level of LUCAT1 in ccRCC cell lines (786-0, Caki1, Caki-2, ACHN, 769-P, and A-498) and one normal kidney cell line (HK-2) by RT-PCR, using $\beta$-actin as a reference gene. We found that the LUCAT1 expression was significantly higher in ccRCC cell lines than in HK-2, which confirmed that LUCAT1 could be a potential oncogene in ccRCC and its expression might correlate with malignancy (Fig. 2D).

\section{Correlation between LUCAT1 expression level and clinical} characteristics

Next, we analyzed the correlation between LUCAT1 expression and the clinical characteristics of ccRCC patients in Sun Yat-sen Memorial Hospital. Statistical analysis revealed that the LUCAT1 expression positively correlated with T stage $(P<0.01)$ and TNM stage $(P$ $<0.01$ ) in 90 ccRCC patients. However, no

Table 3. Correlation between LUCAT1 expression level and clinical characteristics of ccRCC patients. ${ }^{*} \mathrm{P}<0.05$ is considered statistically significant

\begin{tabular}{lcccccc}
\hline Characteristics & number & $\begin{array}{c}\text { Sun Yat-sen set } \\
\text { expression }\end{array}$ & P value & number & $\begin{array}{c}\text { TCGA set } \\
\text { expression }\end{array}$ & P value \\
\hline Gender & & & 0.309 & & & 0.112 \\
Male & 64 & 0.0121 & & 285 & -1.232 & \\
Female & 26 & 0.0208 & & 159 & -1.577 & \\
Age & & & 0.366 & & & 0.169 \\
$<60$ & 58 & 0.0172 & & 200 & -1.514 & \\
$\geq 60$ & 32 & 0.0099 & & 244 & -1.226 & \\
Size (cm) & & & 0.070 & & & 0.142 \\
$<3$ & 26 & 0.0039 & & 409 & -1.401 & \\
$\geq 3$ & 64 & 0.0190 & & 35 & -0.832 & \\
T stage & & & $<0.01^{*}$ & & & $<0.01^{*}$ \\
T1-2 & 70 & 0.0074 & & 273 & -1.735 & \\
T3-4 & 20 & 0.0398 & & 171 & -0.751 & \\
N stage & & & 0.58 & & & 0.246 \\
N0 & 84 & 0.0152 & & 216 & -1.394 & \\
N1 & 6 & 0.0066 & & 16 & -0.454 & \\
NX & & & & 212 & -1.386 & \\
M stage & & & 0.811 & & & $<0.01^{*}$ \\
M0 & 87 & 0.0148 & & 373 & -1.531 & \\
M1 & 3 & 0.0096 & & 71 & -0.437 & \\
TMN stage & & & $<0.01^{*}$ & & & $<0.01^{*}$ \\
I-II & 67 & 0.0073 & & 257 & -1.781 & \\
III-IV & 23 & 0.0358 & & 187 & -0.772 & \\
\hline & & & & & & \\
\hline
\end{tabular}

Fig. 3. LUCAT1 knockdown inhibited ccRCC cell proliferation and colony formation. (A) Subcellular fractionation of LUCAT1 in Caki-2 and 786-0 cells. U6 and GAPDH were used as internal controls. LUCAT1 is predominantly localized in the nucleus. (B) Efficient LUCAT1 knockdown in Caki-2 and 786$\mathrm{O}$ after transfection with siRNAs and negative control. (C) Colony formation ability was inhibited by LUCAT1 knockdown in Caki-2 and 786-0. The histogram shows the average number of surviving clones. (D) LUCAT1 knockdown inhibited proliferation in Caki2 and $786-0$ as determined by CellTiter 96 AQueous One Solution Cell Proliferation Assay. (E) Flow cytometry analysis of cell cycle 48 $\mathrm{h}$ after transfection with siRNAs and negative controls in Caki- 2 and 786-O cells. (F) Western blotting analysis of the expression of cell

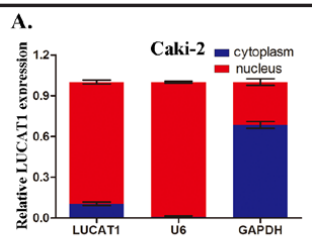

c.
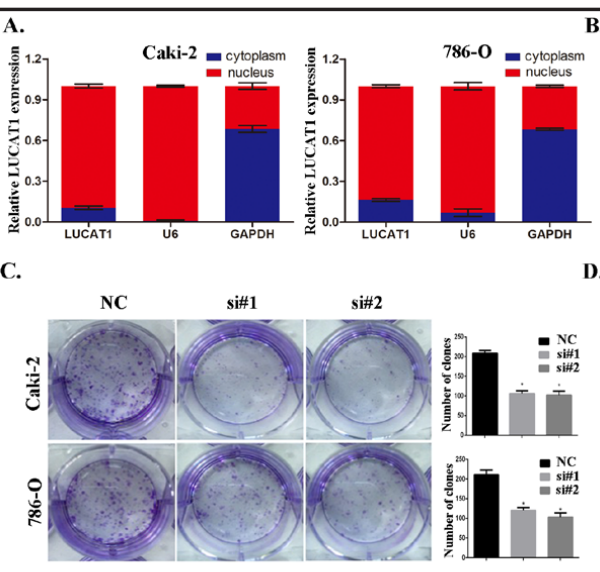

B.
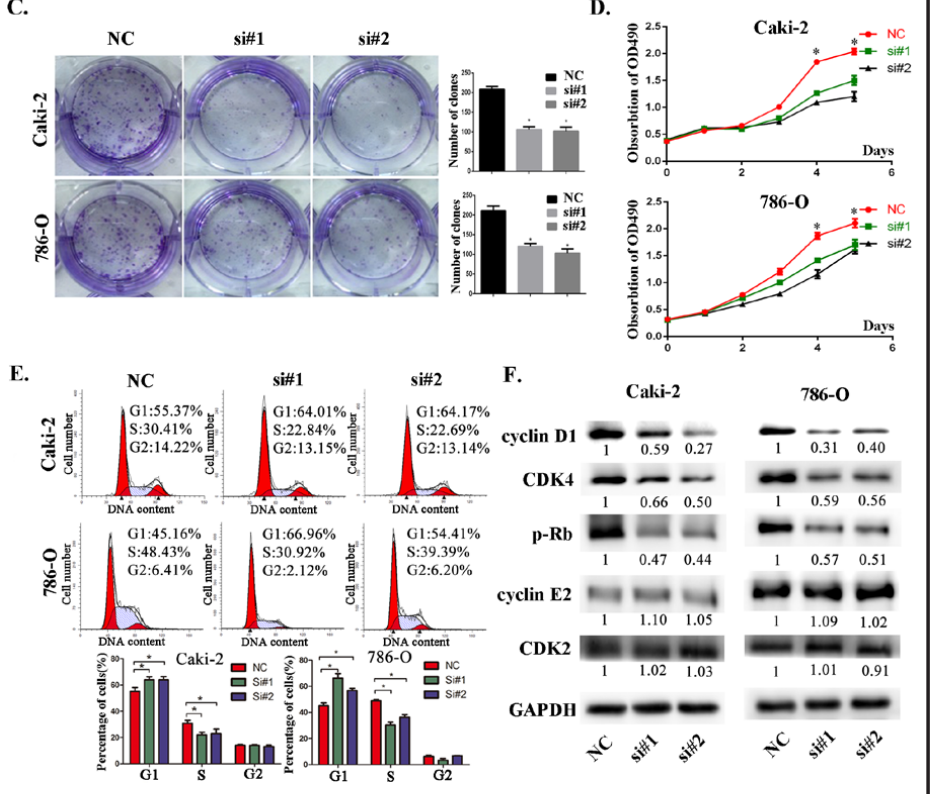
cycle-related proteins. Mean \pm SD is shown $(\mathrm{n}=3) .{ }^{*} \mathrm{P}<0.05$ by Student's $t$ test. 
significant correlation was found between LUCAT1 expression and gender, age, tumor size, $\mathrm{N}$ stage, and $\mathrm{M}$ stage (Table 3).

To further validate the association between LUCAT1 expression level and the clinical characteristics of ccRCC patients, LUCAT1 RNA-Seq expression values and relevant clinical information of 448 TCGA ccRCC patients were downloaded from TCGA and TANRIC websites and statistical analysis was performed. The results showed that LUCAT1 expression significantly correlated with T stage $(P<0.01)$, M stage $(P<0.01)$, and TNM stage $(P<0.01)$ (Table 3).

\section{LUCAT1 expression predicts prognosis of ccRCC patients}

To identify the prognostic value of LUCAT1, we examined the correlation between LUCAT1 expression and prognosis using the Kaplan-Meier method. ccRCC patients in TCGA database were divided into a high-LUCAT1-expression group $(n=224)$ and low-LUCAT1expression group $(n=224)$ by the median expression level. The Kaplan-Meier analysis showed that overall survival and disease-free survival time were significantly shorter in the high-LUCAT1-expression group than in the low group (log-rank $P<0.01$ ) (Fig. 2E and F). To further evaluate the prognostic value of LUCAT1 in ccRCC, we carried out univariate analysis using age, gender, tumor size, T stage, $\mathrm{N}$ stage, M stage, and LUCAT1 expression. The result revealed that LUCAT1 expression was an independent predictor of overall survival (Table 4). Taken together, these data suggested that LUCAT1 might play an important role in ccRCC progression and act as an unfavorable prognostic factor.

\section{LUCAT1 knockdown inhibits proliferation, migration, and invasion of ccRCC cells}

To investigate the subcellular localization of LUCAT1 in ccRCC cells, we isolated total RNA from nuclear and cytosolic fractions of Caki-2 and 786-0. RT-PCR was performed to measure LUCAT1 expression in both fractions. U6 is known to be a nuclear localized RNA and GAPDH, a protein-coding mRNA, was expected in the cytoplasm. The result showed that LUCAT1 was preferentially located in the nucleus (Fig. 3A). To evaluate the role of LUCAT1 in ccRCC progression, siRNAs were designed to knock down LUCAT1. The RT-PCR results showed that LUCAT1 was significantly downregulated by siRNA\#1 (si\#1) and siRNA\#2 (si\#2) in two ccRCC cell lines, Caki-2 and 786-0 (Fig. 3B). Colony formation assays were employed to examine the effect of LUCAT1 on cell growth of ccRCC. The results indicated that LUCAT1 knockdown significantly inhibited the proliferation and colony formation of 786-O and Caki-2 (Fig. 3C and D). The effect of LUCAT1 on the cell cycle was observed by flow cytometry. Compared with the negative control, LUCAT1 knockdown significantly increased the percentage of cells in G1 phase and decreased the percentage of cells in S phase (Fig. 3E). Additionally, apoptosis assay by flow cytometry was employed and no significant difference was found. To support the flow cytometry results, Western blotting was performed to measure the G1/S cell cycle checkpoint-related proteins. The result showed that the expression of cyclin D1, CDK4, and p-Rb was decreased after LUCAT1 knockdown, while cyclin E2 and CDK2 showed no obvious changes (Fig. 3F).

We further evaluated the effects of LUCAT1 on ccRCC cell migration and invasion. The wound-healing assay revealed that LUCAT1 knockdown inhibited cell mobility in both 786-0 and Caki-2 (Fig. 4A). The transwell migration assay demonstrated that downregulation of LUCAT1 significantly reduced the migration of both 786-0 and Caki-2 (Fig. 4B). Furthermore, LUCAT1 knockdown significantly reduced the invasive

Table 4. Univariate and multivariate analysis of factors associated with overall survival in ccRCC. CI, confidence interval; HR, hazard ratio. ${ }^{*} \mathrm{P}<0.05$ is considered statistically significant

\begin{tabular}{|c|c|c|c|c|c|c|}
\hline \multirow{2}{*}{ Variable } & \multicolumn{3}{|c|}{ Univariate } & \multicolumn{3}{|c|}{ Multivariate } \\
\hline & $\mathrm{HR}$ & $95 \% \mathrm{CI}$ & $P$ & $\mathrm{HR}$ & $95 \% \mathrm{CI}$ & $P$ \\
\hline Age $(\geq 60 /<60)$ & 1.813 & $1.304-2.521$ & $<0.01^{*}$ & 1.505 & $1.076-2.105$ & $0.017^{*}$ \\
\hline Gender (male/female) & 0.968 & $0.703-1.334$ & 0.843 & & & \\
\hline Tumor size $(\geq 3 /<3 \mathrm{~cm})$ & 1.538 & $0.833-2.840$ & 0.169 & & & \\
\hline Tumor stage (T3-4/T1-2) & 3.252 & $2.365-4.473$ & $<0.01^{*}$ & 1.989 & $1.378-2.870$ & $<0.01^{*}$ \\
\hline $\mathrm{N}$ stage (N1/N0) & 0.917 & $0.783-1.073$ & 0.278 & & & \\
\hline M stage (M1/M0) & 4.408 & $3.190-6.091$ & $<0.01^{*}$ & 2.794 & $1.932-4.039$ & $<0.01^{*}$ \\
\hline LUCAT1 (high/low) & 1.945 & $1.409-2.685$ & $<0.01 *$ & 1.478 & $1.061-2.059$ & $0.021 *$ \\
\hline
\end{tabular}


potential of ccRCC cells in a transwell invasion assay (Fig. 4B). Thus, these results suggested that LUCAT1 knockdown inhibited the migration and invasive ability of ccRCC cells.

\section{LUCAT1 overexpression promotes proliferation, migration, and invasion of ccRCC cells}

We overexpressed LUCAT1 in ccRCC cells by lentivirus vector-mediated overexpression. The expression level of LUCAT1 in 786-0 and Caki-2 was significantly increased after transfection with pLV-LUCAT1 compared with the negative control (pLV-NC) (Fig. 5 A). Colony formation assays were employed to examine the growth of ccRCC cells. The results indicated that LUCAT1 overexpression significantly promoted the proliferation and colony formation of 786-O and Caki-2 (Fig. 5B and C). Flow cytometry analysis showed that LUCAT1 overexpression significantly increased the percentage of cells in $S$ phase and decreased the percentage of cells in G1 phase (Fig. 5D). The transwell migration and invasion assays demonstrated that LUCAT1 overexpression significantly promoted the migration and invasive ability in both 786-0 and Caki-2 (Fig. 5E). Together, these results showed that LUCAT1 overexpression promoted proliferation, migration, and invasion of ccRCC cells.

Fig. 4. LUCAT1 knockdown inhibited migration and invasion of ccRCC cells. (A) Woundhealing assay in Caki-2 and 786-0 after transfection with siRNAs and negative controls. (B) Representative images of cell migration or invasion across the membrane with LUCAT1 knockdown. The histograms show the average number of migrated or invasive cells per field calculated from 5 representative fields. ${ }^{*} \mathrm{P}<0.05$ by Student's t test.

Fig. 5. LUCAT1 overexpression promoted proliferation, migration, and invasion of ccRCC cells. (A) LUCAT1 overexpression in Caki2 and 786-0 after transfection with pLV-LUCAT1 and pLV-NC. (B) Colony formation after LUCAT1 overexpression in Caki-2 and 786-0. The histogram shows the average number of the surviving clones. (C) LUCAT1 overexpression promoted proliferation in Caki2 and 786-0 as determined by CellTiter 96 AQueous One Solution Cell Proliferation Assay. (D) Flow cytometry analysis of the cell cycle with LUCAT1 overexpression. (E)

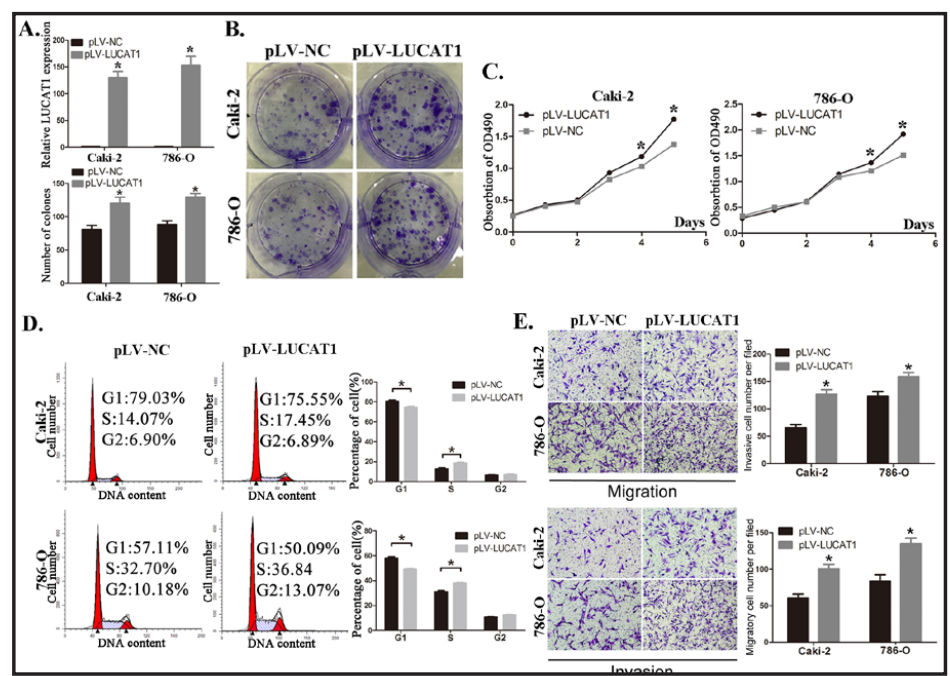

Representative images of cell migration or invasion across the membrane with LUCAT1 overexpression. The histograms show the average number of migrated or invasive cells per field calculated from 5 representative fields. Mean \pm SD is shown $(n=3)$. ${ }^{*} \mathrm{P}<0.05$ by Student's t test.

\section{KARGER}




\section{Cellular Physiology Cell Physiol Biochem 2018;48:891-904 \begin{tabular}{l|l|l|} 
DOI: 10.1159/000491957 & $\begin{array}{l}\text { () 2018 The Author(s). Published by S. Karger AG, Basel } \\
\text { www.karger.com/cpb }\end{array}$
\end{tabular}

LUCAT1 regulates the progression of ccRCC through the AKT/GSK-3 $\beta$ signaling pathway

In order to explore the possible molecular mechanism of LUCAT1's effects, we performed gene set enrichment analysis (GSEA) to identify associated pathways in ccRCC. We compared the gene expression profile between high LUCAT1 expression M1 ccRCC patients with low LUCAT1 expression M0 ccRCC patients in TCGA database. Several cancer-related pathways such as the NF- $\kappa$ B pathway, AKT pathway, and P53 pathway were enriched (Fig. 6A). We performed Western blotting to analyze key proteins of these pathways, and the results showed that p-AKT expression was significantly decreased after LUCAT1 knockdown, while P53 and NF-kB showed no significant change. We further examined the key downstream components of the AKT pathway, including p-GSK-3 $\beta, \beta$-catenin, and p-mTOR. The results showed that p-GSK-3 $\beta$ expression was significantly increased after LUCAT1 knockdown (Fig. 6B). A similar result was found in the LUCAT11 overexpression experiment (Fig. 6C). In order to confirm the important role of the AKT pathway in cCRCC, we overexpressed LUCAT1 in ccRCC cells and concomitantly inhibited the AKT pathway using LY294002. The result showed that inhibition of the AKT pathway significantly reduced the effects of LUCAT1 on the invasive ability of ccRCC cells (Fig. 6D). Therefore, these results suggested that LUCAT1 regulated the progression of ccRCC through the AKT/GSK- $3 \beta$ signaling pathway.

\section{LUCAT1 is regulated by CXCL2 in cCRCC cells}

Next, we investigated the possible mechanisms controlling LUCAT1 expression in ccRCC cells. Previous research suggested that chemokines or cytokines released by immune cells could regulate the expression of IncRNAs in cancer cells [18]. We performed a computational screen by analyzing the correlation between LUCAT1 and chemokines or cytokines using TANRIC. We found that LUCAT1 expression was significantly correlated with chemokine CXCL2 and CXCL8 and cytokine IL-6 mRNA expression in ccRCC tissues (Fig. 7A). We next determined whether LUCAT1 expression in ccRCC cells was mediated by CXCL2, CXCL8, or IL-6. Caki-2 and 786-0 were exposed to CXCL2, CXCL8, and IL-6 at different concentrations for $48 \mathrm{~h}$. The results showed that CXCL2 significantly increased the expression of LUCAT1 in ccRCC cells (Fig. 7B). No significant difference was found with CXCL8 or IL-6 stimulation. We then treated Caki-2 and 786-0 with $50 \mathrm{ng} / \mathrm{ml} \mathrm{CXCL2}$ for various times and the results also

Fig. 6. LUCAT1 regulated the progression of ccRCC through the AKT/GSK-3 $\beta$ signaling pathway. (A) GSEA was performed to identify associated pathways in ccRCC. (B) Western blotting analysis of the expression of associated pathway proteins in ccRCC with LUCAT1 knockdown. (C) Western blotting analysis of the expression of associated pathway proteins in ccRCC with LUCAT1 overexpression. (D). Transwell invasion assay with LUCAT1 overexpression and AKT inhibition in ccRCC. ${ }^{*} \mathrm{P}<0.05$ by Student's $\mathrm{t}$ test.

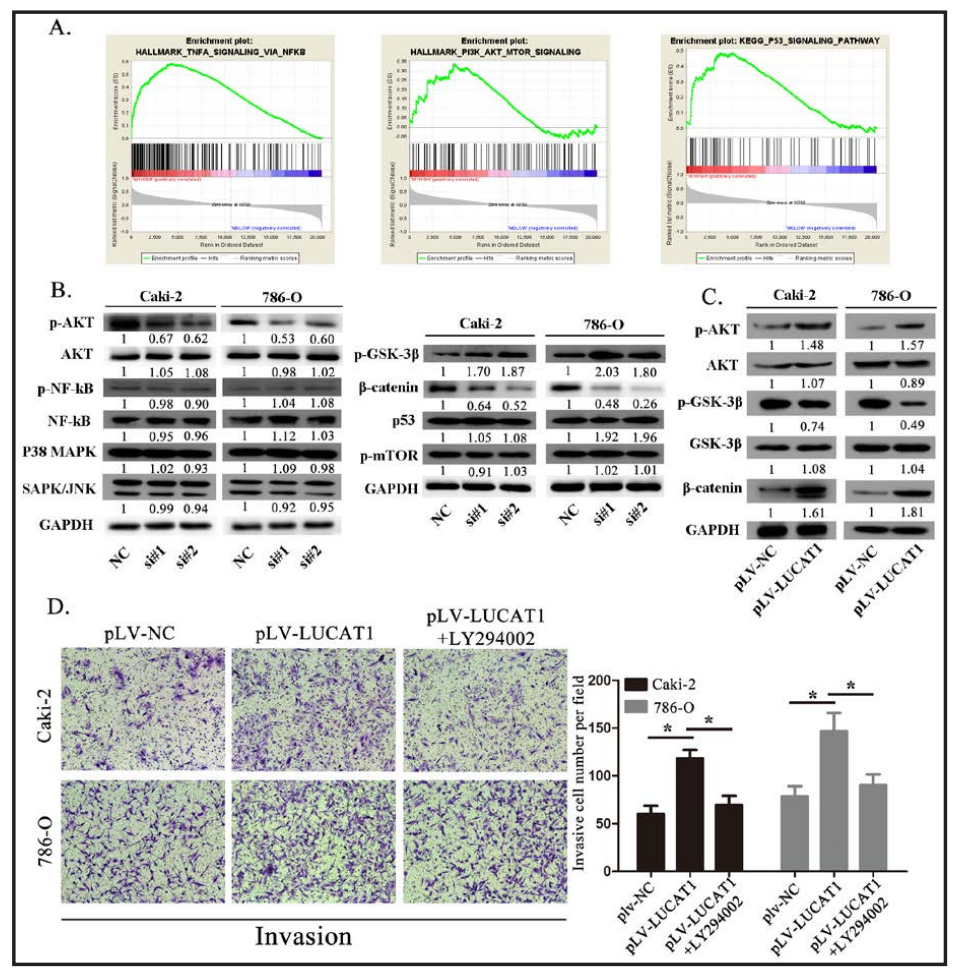


Fig. 7. LUCAT1 overexpression was induced by chemokine CXCL2. (A) Computational analysis of the correlation between LUCAT1 and chemokines or cytokines in ccRCC tissues (Pearson's correlation test, ${ }^{*} \mathrm{P}<0.05$ ). (B) LUCAT1 expression in ccRCC cells after $48 \mathrm{~h}$ of chemokine or cytokine stimulation at different concentrations. Mean \pm SD is shown $(\mathrm{n}=3)$ (Student's $\mathrm{t}$ test, ${ }^{*} \mathrm{P}<0.05$ ). (C) LUCAT1 expression in ccRCC cells after CXCL2 stimulation at different time points. Mean \pm SD is shown ( $\mathrm{n}=3$ ) (Student's t test, ${ }^{*} \mathrm{P}<0.05$ ). (D) Western blotting analysis of the influence of LUCAT1 on CXCL2 expression in ccRCC cells. (E) Gene expression profiling and interactive analysis of CXCL2 expression in ccRCC tissues and normal tissues (Student's $\mathrm{t}$ test, $\left.{ }^{*} \mathrm{P}<0.05\right)$. (F) Kaplan-Meier analysis of overall survival in highCXCL2-expression and low-CXCL2-

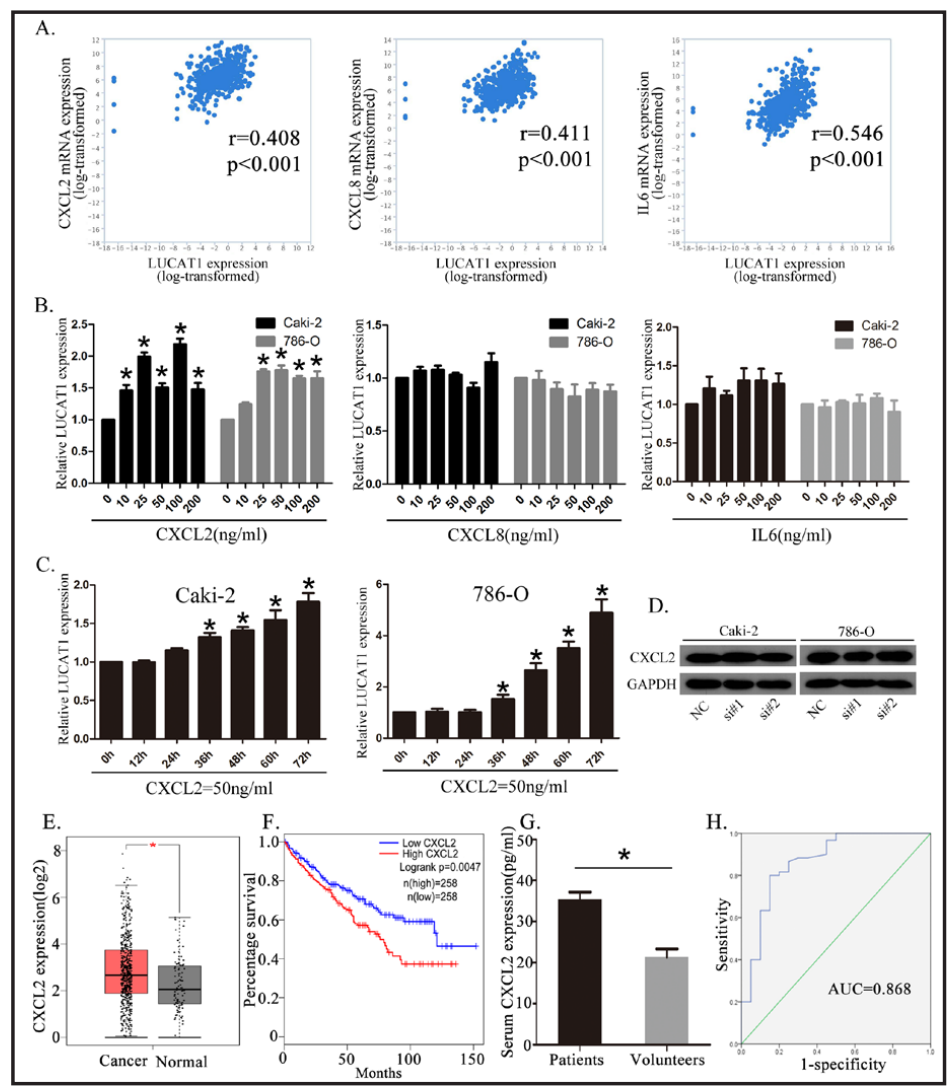
expression ccRCC patients. (G) ELISA detected the serum CXCL2 level in ccRCC patients $(n=60)$ and healthy volunteers $(n=20)$ (Student's t test, $\left.{ }^{*} \mathrm{P}<0.05\right)$. (H) ROC curve analysis of the diagnostic value of serum CXCL2 in ccRCC.

showed that CXCL2 significantly increased the expression of LUCAT1 in ccRCC cells (Fig. 7C). However, LUCAT1 knockdown did not make a difference in CXCL2 expression in ccRCC cells (Fig. 7D). In addition, we analyzed CXCL2 expression between ccRCC tissues and normal tissues using gene expression profiling and interactive analysis (GEPIA). We found that CXCL2 was significantly upregulated in ccRCC tissues ( $\mathrm{n}=523)$ compared with normal tissues (n $=100$ ) (Fig. 7E). The Kaplan-Meier survival analysis revealed that ccRCC patients with higher CXCL2 expression have a shorter

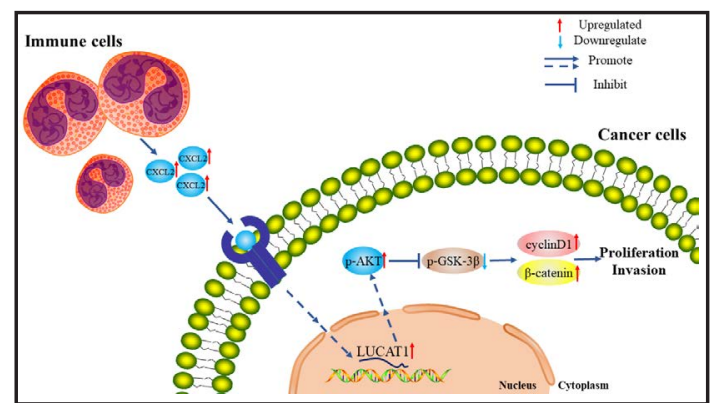

Fig. 8. A schematic representation of possible mechanism for CXCL2- and LUCAT1-mediated proproliferation and pro-invasion in ccRCC. overall survival time (Fig. 7F). We also analyzed the serum CXCL2 level in ccRCC patients $(n=60)$ and healthy volunteers $(n=20)$, and found that serum CXCL2 expression in CCRCC patients was significantly higher than in healthy volunteers (Fig. 7G). ROC curve analysis revealed that serum CXCL2 level might be a diagnostic biomarker (AUC $=0.868$ ) for ccRCC (Fig. $7 \mathrm{H}$ ). In summary, these results showed that the expression of LUCAT1 was regulated by CXCL2 in ccRCC cells.

These data, along with the above knockdown and overexpression experiments, indicated that CXCL2 in tumor microenvironments, synthesized and secreted by immune cells, induced LUCAT1 expression and promoted proliferation and invasion in ccRCC (Fig. 8). 


\section{Discussion}

It has been demonstrated that the human transcriptome comprises not only proteincoding RNAs but also a large number of ncRNAs, including lncRNAs [19]. IncRNAs are emerging as functional regulators in various cancer processes, including proliferation, invasion, and metastasis $[8,20,21]$. However, few lncRNAs have been well characterized in ccRCC. In this study, we used microarray analysis and found that 943 lncRNAs are upregulated and 611 lncRNAs are downregulated (fold change $>2$ and $P<0.01$ ) in ccRCC.

Among these IncRNAs, LUCAT1, located at chromosome 5q14.3, has been found to be associated with poor prognosis in non-small cell lung cancer and promotes cell proliferation via regulating p21 and p57 expression [22]. In this study, we found that LUCAT1 was overexpressed in ccRCC tissues and cell lines compared with adjacent normal tissues and a normal kidney epithelial cell line. We also analyzed the association between LUCAT1 expression and clinical characteristics of ccRCC patients. We found that the LUCAT1 expression significantly correlated with $\mathrm{T}$ stage, $\mathrm{M}$ stage, and TNM stage. The prognostic value of LUCAT1 in ccRCC was also analyzed using the Kaplan-Meier method and we found that LUCAT1 expression was significantly associated with the overall survival and diseasefree survival of ccRCC patients. ccRCC patients with high LUCAT1 expression had poorer survival than those with low LUCAT1 expression. To the best of our knowledge, this is the first report showing that LUCAT1 might be a predictor of survival in ccRCC patients.

The unlimited proliferation of cancer cells is often due to disorders of the cell cycle $[23,24]$. Our research team previously reported that knockdown of lncRNA AATBC resulted in inhibited bladder cancer cell proliferation through cell cycle arrest [25]. The G1/S cell cycle checkpoint, the major regulatory checkpoint, controls progression of cells through the restriction point into the S-phase [26]. The cyclin D1-CDK4 complex contributes to the progression of cells through the G1/S cell cycle checkpoint via increasing phosphorylation of $\mathrm{Rb}$ [27]. In the present study, the cell proliferation assay, colony formation assay, and flow cytometry analysis demonstrated that LUCAT1 promoted ccRCC cell proliferation and increased the percentage of cells in S phase. Western blot provided molecular evidence supporting these observations. The results showed that the expression of cyclin D1, CDK4, and $\mathrm{p}-\mathrm{Rb}$ was decreased after LUCAT1 knockdown. Therefore, the most likely mechanism underlying the proliferation regulation involves inhibiting the cyclin D1-CDK4 complex, and then ultimately inducing cell cycle arrest at the G1 phase.

As LUCAT1 expression significantly correlated with T stage and M stage, we suspected that LUCAT1 could play an important role in ccRCC metastasis. We therefore determined whether LUCAT1 expression influenced ccRCC migration and invasion. The wound-healing and transwell migration and invasion assays demonstrated that LUCAT1 promoted migration and invasive ability of ccRCC cells. These results suggested that LUCAT1 could promote ccRCC metastasis by affecting migration and invasion.

Aberrant AKT and Wnt pathways contribute to cell proliferation and metastasis in many kinds of cancers $[28,29]$. The interaction between the two pathways through the AKT/GSK$3 \beta$ axis has been found to participate in the progression of ccRCC [30]. AKT phosphorylation can inhibit the activity of GSK-3 $\beta$, which plays a vital role in $\beta$-catenin phosphorylation and degradation, and activate the transcription of downstream target oncogenes such as cyclin D1 [31, 32]. In our research, LUCAT1 knockdown reduced the expression level of p-AKT and activated GSK-3 $\beta$, resulting in the downregulation of $\beta$-catenin and cyclin D1. Moreover, LUCAT1 overexpression significantly promoted AKT phosphorylation and then inhibited the activity of GSK-3 $\beta$, and ultimately promoted the progression of ccRCC. Therefore, these results suggested that the molecular mechanism underlying LUCAT1's ability to induce proliferation and invasion in ccRCC might depend on the activation of the AKT/GSK-3 $\beta$ signaling pathway.

We further explored the possible mechanisms underlying how LUCAT1 expression is dysregulated in ccRCC. Computational screen and stimulation assay revealed that the chemokine CXCL2 induced LUCAT1 expression in ccRCC cells. In addition, serum CXCL2 expression in ccRCC patients is significantly higher than in healthy volunteers and ccRCC

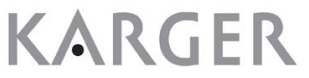


patients with higher CXCL2 expression have a shorter overall survival time. To the best of our knowledge, CXCL2 is mainly synthesized and secreted by macrophages or other immune cells that could promote cancer progression and metastasis by releasing a variety of chemokines [33, 34]. Therefore, one possible mechanism regulating LUCAT1 expression in ccRCC is that CXCL2 in the tumor microenvironment, synthesized and secreted by macrophages or other immune cells, bound to a specific transmembrane receptor on ccRCC cells and induced LUCAT1 expression. The upregulation of LUCAT1 promoted the phosphorylation of AKT and then inhibited the activity of GSK-3 $\beta$. On one hand, inhibition of GSK- $3 \beta$ reduced the degradation of $\beta$-catenin and promoted invasion of ccRCC. On the other hand, inhibition of GSK- $3 \beta$ reduced the degradation of cell cycle-related proteins, such as cyclin D1, and facilitated proliferation of ccRCC. Our study highlights the importance of the tumor microenvironment in the progression of ccRCC and it is worthy of further research.

In conclusion, we found that LUCAT1 was upregulated in ccRCC tissues and renal cancer cell lines, and significantly correlated with malignant stage and prognosis in ccRCC. LUCAT1 promoted proliferation and invasion of ccRCC cells partly through the AKT/GSK- $3 \beta$ signaling pathway. We also revealed that LUCAT1 overexpression was induced by the chemokine CXCL2. These results suggest that the CXCL2/LUCAT1/AKT/GSK-3 $\beta$ axis plays a critical role in promoting the progression of ccRCC, and might be a useful diagnostic biomarker and therapeutic target in ccRCC.

\section{Acknowledgements}

This work was supported by grants from the National Natural Science Foundation of China (Nos. 81672534 and 81472388), grant [2013]163 from the Key Laboratory of Malignant Tumor Molecular Mechanism and Translational Medicine of Guangzhou Bureau of Science and Information Technology, grant KLB09001 from the Key Laboratory of Malignant Tumor Gene Regulation and Target Therapy of Guangdong Higher Education Institutes, and grants from the Guangdong Science and Technology Department (Nos. 2013 B021800110 and 2015B050501004).

This study was performed according to the recommendations in the Guide for the Chinese Ethics Review Committees. The protocol was approved by the Ethics Committee of Sun Yat-sen Memorial Hospital. Written informed consent was obtained from each subject.

\section{Disclosure Statement}

The authors declare that they have no competing interests.

\section{References}

1 Siegel RL, Miller KD, Jemal A: Cancer statistics, 2016 CA Cancer J Clin 2016;66:7-30.

-2 Shuch B, Amin A, Armstrong AJ, Eble JN, Ficarra V, Lopez-Beltran A, Martignoni G, Rini BI, Kutikov A: Understanding pathologic variants of renal cell carcinoma: distilling therapeutic opportunities from biologic complexity. Eur Urol 2015;67:85-97.

-3 Capitanio U, Montorsi F: Renal cancer. Lancet 2016;387:894-906.

4 Gupta K, Miller JD, Li JZ, Russell MW, Charbonneau C: Epidemiologic and socioeconomic burden of metastatic renal cell carcinoma (mRCC): a literature review. Cancer Treat Rev 2008;34:193-205.

Cohen HT, McGovern FJ: Renal-cell carcinoma. N Engl J Med 2005;353:2477-2490.

Vance KW, Ponting CP: Transcriptional regulatory functions of nuclear long noncoding RNAs. Trends Genet 2014;30:348-355.

7 Beermann J, Piccoli MT, Viereck J, Thum T: Non-coding RNAs in Development and Disease: Background, Mechanisms, and Therapeutic Approaches. Physiol Rev 2016;96:1297-1325.

8 Hirata H, Hinoda Y, Shahryari V, Deng G, Nakajima K, Tabatabai ZL, Ishii N, Dahiya R: Long Noncoding RNA MALAT1 Promotes Aggressive Renal Cell Carcinoma through Ezh2 and Interacts with miR-205 Cancer Res 2015;75:1322-1331. 


\section{Cellular Physiology Cell Physiol Biochem 2018;48:891-904 \begin{tabular}{l|l} 
and Biochemistry Published 10.1159/000491957 & $\begin{array}{l}\text { (c) } 2018 \text { The Author(s). Published by S. Karger AG, Basel } \\
\text { www.karger.com/cpb }\end{array}$
\end{tabular}}

Zheng et al.: LUCAT1 Promoted Proliferation and Invasion in Ccrcc

9 Li J, Wang J, Chen Y, Li S, Jin M, Wang H, Chen Z, Yu W: LncRNA MALAT1 exerts oncogenic functions in lung adenocarcinoma by targeting miR-204. Am J Cancer Res 2016;6:1099-1107.

10 Li L, Chen H, Gao Y, Wang YW, Zhang GQ, Pan SH, Ji L, Kong R, Wang G, Jia YH, Bai XW, Sun B: Long Noncoding RNA MALAT1 Promotes Aggressive Pancreatic Cancer Proliferation and Metastasis via the Stimulation of Autophagy. Mol Cancer Ther 2016;15:2232-2243.

11 Lu L, Zhu G, Zhang C, Deng Q, Katsaros D, Mayne ST, Risch HA, Mu L, Canuto EM, Gregori G, Benedetto C, Yu H: Association of large noncoding RNA HOTAIR expression and its downstream intergenic CpG island methylation with survival in breast cancer. Breast Cancer Res Treat 2012;136:875-883.

12 Quagliata L, Matter MS, Piscuoglio S, Arabi L, Ruiz C, Procino A, Kovac M, Moretti F, Makowska Z, Boldanova T, Andersen JB, Hammerle M, Tornillo L, Heim MH, Diederichs S, Cillo C, Terracciano LM: Long noncoding RNA HOTTIP/HOXA13 expression is associated with disease progression and predicts outcome in hepatocellular carcinoma patients. Hepatology 2014;59:911-923.

13 Kong R, Zhang EB, Yin DD, You LH, Xu TP, Chen WM, Xia R, Wan L, Sun M, Wang ZX, De W, Zhang ZH: Long noncoding RNA PVT1 indicates a poor prognosis of gastric cancer and promotes cell proliferation through epigenetically regulating p15 and p16. Mol Cancer 2015;14:82.

14 Wang F, Li X, Xie X, Zhao L, Chen W: UCA1, a non-protein-coding RNA up-regulated in bladder carcinoma and embryo, influencing cell growth and promoting invasion. FEBS Lett 2008;582:1919-1927.

15 Thai P, Statt S, Chen CH, Liang E, Campbell C, Wu R: Characterization of a novel long noncoding RNA, SCAL1, induced by cigarette smoke and elevated in lung cancer cell lines. Am J Respir Cell Mol Biol 2013;49:204211.

16 Li J, Han L, Roebuck P, Diao L, Liu L, Yuan Y, Weinstein JN, Liang H: TANRIC: An Interactive Open Platform to Explore the Function of IncRNAs in Cancer. Cancer Res 2015;75:3728-3737.

17 Paner GP, Stadler WM, Hansel DE, Montironi R, Lin DW, Amin MB: Updates in the Eighth Edition of the Tumor-Node-Metastasis Staging Classification for Urologic Cancers. Eur Urol 2018;73:560-569.

18 Sun C, Xue L, Zhu Z, Zhang F, Yang R, Yuan X, Jia Z, Liu Q: Insights from IncRNAs Profiling of MIN6 Beta Cells Undergoing Inflammation. Mediators Inflamm 2016;2016:9275106.

19 Wilusz JE, Sunwoo H, Spector DL: Long noncoding RNAs: functional surprises from the RNA world. Genes Dev 2009;23:1494-1504.

20 Huarte M: The emerging role of IncRNAs in cancer. Nat Med 2015;21:1253-1261.

-21 Raveh E, Matouk IJ, Gilon M, Hochberg A: The H19 Long non-coding RNA in cancer initiation, progression and metastasis - a proposed unifying theory. Mol Cancer 2015;14:184.

22 Sun Y, Jin SD, Zhu Q Han L, Feng J, Lu XY, Wang W, Wang F, Guo RH: Long non-coding RNA LUCAT1 is associated with poor prognosis in human non-small lung cancer and regulates cell proliferation via epigenetically repressing p21 and p57 expression. Oncotarget 2017;8:28297-28311.

23 Bell JL, Malyukova A, Kavallaris M, Marshall GM, Cheung BB: TRIM16 inhibits neuroblastoma cell proliferation through cell cycle regulation and dynamic nuclear localization. Cell Cycle 2013;12:889-898.

24 Liu X, Yang WT, Zheng PS: Msi1 promotes tumor growth and cell proliferation by targeting cell cycle checkpoint proteins p21, p27 and p53 in cervical carcinomas. Oncotarget 2014;5:10870-10885.

25 Zhao F, Lin T, He W, Han J, Zhu D, Hu K, Li W, Zheng Z, Huang J, Xie W: Knockdown of a novel lincRNA AATBC suppresses proliferation and induces apoptosis in bladder cancer. Oncotarget 2015;6:1064-1078.

26 Kastan MB, Bartek J: Cell-cycle checkpoints and cancer. Nature 2004;432:316-323.

27 Malumbres M: Cyclin-dependent kinases. Genome Biol 2014;15:122.

28 Manning BD, Cantley LC: AKT/PKB signaling: navigating downstream. Cell 2007;129:1261-1274.

29 Zhan T, Rindtorff N, Boutros M: Wnt signaling in cancer. Oncogene 2017;36:1461-1473.

-30 Chen Q Yang D, Zong H, Zhu L, Wang L, Wang X, Zhu X, Song X, Wang J: Growth-induced stress enhances epithelial-mesenchymal transition induced by IL-6 in clear cell renal cell carcinoma via the Akt/GSK-3beta/ beta-catenin signaling pathway. Oncogenesis 2017;6:e375.

-31 Takahashi-Yanaga F, Sasaguri T: GSK-3beta regulates cyclin D1 expression: a new target for chemotherapy. Cell Signal 2008;20:581-589.

-32 Diehl JA, Cheng M, Roussel MF, Sherr CJ: Glycogen synthase kinase-3beta regulates cyclin D1 proteolysis and subcellular localization. Genes Dev 1998;12:3499-3511.

33 Chen J, Yao Y, Gong C, Yu F, Su S, Chen J, Liu B, Deng H, Wang F, Lin L, Yao H, Su F, Anderson KS, Liu Q, Ewen ME, Yao X, Song E: CCL18 from tumor-associated macrophages promotes breast cancer metastasis via PITPNM3. Cancer Cell 2011;19:541-555.

-34 Wan S, Zhao E, Kryczek I, Vatan L, Sadovskaya A, Ludema G, Simeone DM, Zou W, Welling TH: Tumorassociated macrophages produce interleukin 6 and signal via STAT3 to promote expansion of human hepatocellular carcinoma stem cells. Gastroenterology 2014;147:1393-1404. 